\title{
GLAD!
}

Revue sur le langage, le genre, les sexualités

$07 \mid 2019$

Varia

\section{Entretiens aux tarots/Johanna Rocard}

Tarot Talks/Johanna Rocard

\section{Ophélie Naessens et Johanna Rocard}

\section{(e) OpenEdition}

Journals

Édition électronique

URL : http://journals.openedition.org/glad/1707

DOI : $10.4000 /$ glad. 1707

ISSN : 2551-0819

Éditeur

Association GSL

Référence électronique

Ophélie Naessens et Johanna Rocard, «Entretiens aux tarots/Johanna Rocard », GLAD! [En ligne], 07 | 2019, mis en ligne le 05 décembre 2019, consulté le 17 décembre 2020. URL : http:// journals.openedition.org/glad/1707 ; DOI : https://doi.org/10.4000/glad.1707

Ce document a été généré automatiquement le 17 décembre 2020.

\section{(c) (i) (9)}

La revue GLAD! est mise à disposition selon les termes de la Licence Creative Commons Attribution Pas d'Utilisation Commerciale - Pas de Modification 4.0 International. 


\section{Entretiens aux tarots/Johanna Rocard}

Tarot Talks/Johanna Rocard

Ophélie Naessens et Johanna Rocard

Cet entretien est le premier d'une série initiée dans le cadre d'une enquête plus vaste menée auprès de plasticiennes qui emploient des techniques divinatoires dans leur pratique artistique. Ces interviews prennent la forme d'un tirage de tarots, non pas dans la perspective d'une projection du futur mais davantage dans celle d'un état des lieux de la situation artistique actuelle. Le premier entretien s'est déroulé à Rennes le 18 mars 2019 avec Johanna Rocard ${ }^{1}$.

\section{Qu'est-ce que les pratiques divinatoires peuvent} apporter à l'art ? (question de la consultante). La coupe 
Première carte : I'Ermite

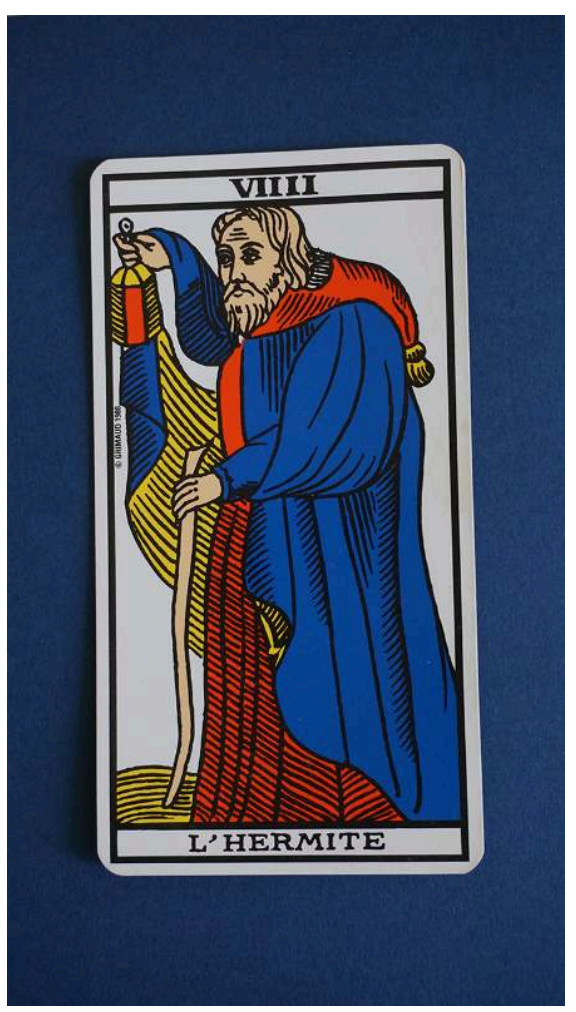

Cartes tirées pendant l'entretien.

Johanna Rocard

Ophélie Naessens. Un homme porte une lumière en marchant. C'est une figure souvent liée dans les histoires à un·e oracle, à quelqu'un·e qui vient transmettre une parole de l'ordre du magique ou du divin. Je trouve curieux que la première carte montre un homme alors que, depuis le début de cette enquête sur les pratiques artistiques divinatoires, ce sont plutôt des femmes que je rencontre (Tatiana Karl Pez, Violaine Lochu, Milady Renoir, Camilla Graff Junior, etc.). Ces figures de femmes renvoient à celle de l'oracle; des femmes qui, d'une certaine manière, réactivent le pouvoir des sibylles antiques. Aussi, dans ces pratiques divinatoires, il y a toujours des objets, pas toujours des cartes de tarots, des objets qui circulent entre les mains du.de la participant.e et celles de la performeuse.

Johanna Rocard. C'est justement quelque chose qui m'intéresse dans la mesure où je m'interroge sur mon utilisation du livre de Jodorowski (La voie du tarot. 2004). Si ce livre est un état des lieux interprétatif riche, cela me pose question de me référer au livre d'un homme, proposant une interprétation très genrée, alors que je suis traversée par ces expériences de la divination plutôt à travers des pratiques et des écrits de femmes. La question du genre dans le tarot est très présente dans mon entourage. Comment négocier dans la projection les relations entre figures masculines et féminines? Et, surtout, comment se défaire des interprétations genrées attachées à cet outil plutôt occidental et marqué par des centaines d'années de stratifications d'une société patriarcale? Par exemple, pourquoi l'Empereur, la figure la plus masculine, la plus virile, représenterait-elle la stabilité, la force? Les figures féminines sont plus ambivalentes, mais elles sont aussi très puissantes. Je n'ai pas vu cette carte comme une figure de la question, l'ermite comme oracle. J'envisage l'ermite sous les traits de la solitude comme si c'était l'art, la figure solitaire. C'est comme un point de départ: où en sommes-nous dans l'art? L'art serait un 
personnage dans le repli. Mais les deux lectures fonctionnent ensemble puisque c'est une guide, celui.celle qui a la lumière.

O.N. Chez l'ermite, ou l'anachorète du Moyen-âge, ces deux visages existent: la contemplation positive, une méditation qui entraine vers un niveau spirituel supérieur, et le repli sur soi.

$J . R$. L'ermite, c'est aussi la figure du passage. Nous serions aujourd'hui dans l'art dans un état de passage, un état de crise représenté par ce personnage seul en marche.

O.N. C'est aussi un personnage qui laisse tomber des choses, qui se défait des biens matériels pour s'engager dans une quête spirituelle.

$J . R$. Cette carte «symbolise une crise à laquelle il faut se donner, un changement profond auquel il convient de faire face ». Qui sera lela guide durant cette phase de crise? Est-ce qu'il n'y a pas besoin de changer un système? Trouver d'autres mécanismes, modifier quelque chose d'essentiel dans la manière dont le système artistique fonctionne. La carte nous signifie qu'il y a un changement à apporter.

Deuxième carte : la Papesse

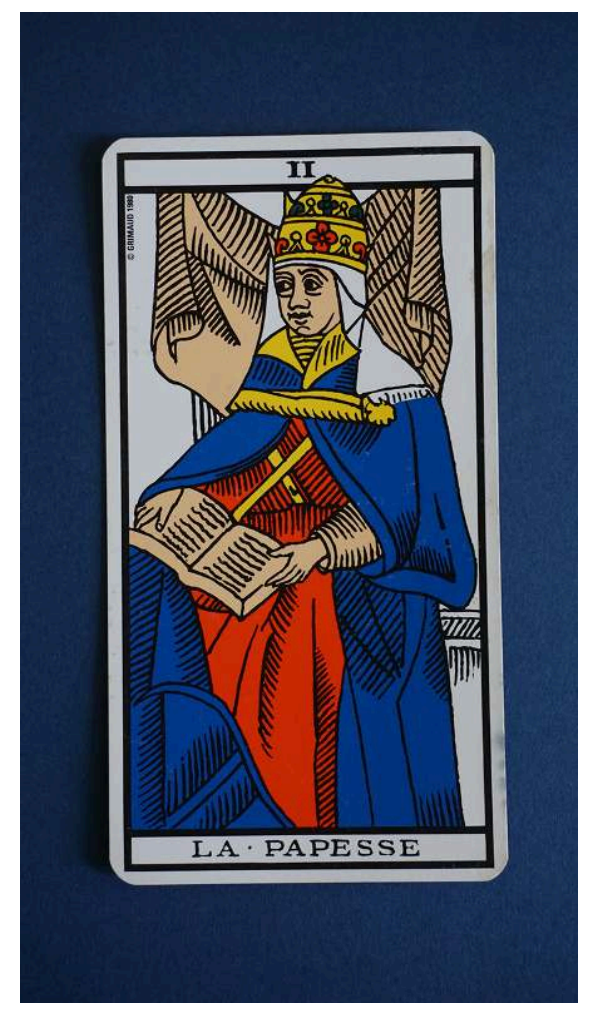

O.N. La carte me renvoie à ces figures de femmes qui ont une prestance, qui se trouvent dans des situations de hiérarchie et, en même temps, je n'arrive pas à la voir comme quelqu'un·e de bienveillant.e. Elle a un livre dans la main, c'est aussi une figure de savoir. Et, plus littéralement, elle lit, comme des performeuses lisant les cartes.

$J . R$. Sur ce jeu en particulier, il lui manque un détail. Normalement, elle est assise sur un œuf. C'est la figure de la gestation et de l'accumulation des savoirs. Nos premières figures sont donc résolument solitaires. Elle se remplit de savoir, elle se nourrit et attend quelque chose. Dans l'interprétation jodorowskienne, c'est la mère froide, sans sexualité. Je trouve cela particulièrement ennuyant que la femme qui sache soit la femme sans sexualité, et je crois au contraire que d'autres choses se disent dans cette 
carte. Nos deux premières figures ont la même combinaison de vêtements ; les deux ont une cape bleue à l'intérieur rouge. Deux personnages associée's à l'idée de sagesse, et pourtant, à l'intérieur... Il y a cette ouverture béante rouge en forme de vagin. Mais c'est une lecture qui revient constamment chez les consultante's; la femme froide, distante, la mauvaise mère, la femme sans tendresse. Cette lecture est donc profondément imprégnée culturellement, et appelle à être déconstruite.

O.N. En cela, la pratique du tarot dans l'art pourrait induire des pistes pour questionner les lectures genrées dans notre culture visuelle.

$J . R$. Elle couve un œuf, " elle le couve dans une grande exigence et dans une haute solitude ». C'est de cette exigence et de cette solitude que peut émerger quelque chose. Un œuf attend d'éclore. Dans ce premier tirage, nous avons deux personnages solitaires en transition. On pourrait imaginer l'art contemporain comme ce personnage solitaire qui serait dans un besoin de transition, à la recherche de nouvelles manières de faire. On peut observer aujourd'hui dans certains lieux d'art, par exemple au Magasin à Grenoble, au 49 Nord 6 Est - Frac Lorraine, une volonté d'ouvrir leurs portes à d'autres disciplines que celles artistiques, de s'autoriser à être politiques. Et ce sont justement ces lieux qui convoquent les pratiques divinatoires, ésotériques.

O.N. D'une certaine manière, en se réappropriant la question des croyances et des cultures populaires, ces lieux d'art montrent qu'ils sont prêts à parler le même langage que tout le monde. Les éléments d'imaginaire convoqués sont collectivement partagés, nous les avons en commun autant avec nos grand-mères qu'avec nos voisines. Et, paradoxalement, ils renvoient à quelque chose de l'ordre du code, d'un système de communication partagé par des « initié.e.s » faisant partie d'un cercle.

$J . R$. Je me pose justement la question du cercle dans ces pratiques ésotériques. Comment le cercle se crée-t-il ? Quels en sont les codes? Qui est exclu? Qui est inclus? Dans tous les cas, ce ne sont pas ceux.celles qui sont attenduees dans le monde de l'art. Lorsque tu convoques ces pratiques, tu es par exemple susceptible d'accueillir des personnes du domaine somatique, médical. Mais se pose toujours la question de la marge, jusqu'où accepte-t-on ceux.celles qui sont à la marge du champ artistique au cœur de la recherche (artistique et scientifique)?

O.N. C'est aussi ce qui se joue dans un certain nombre de workshops collaboratifs: on recrée un cercle dans lequel se rejouent des rapports d'inclusion et d'exclusion; qui parle? Qui écoute ? Qui est à l'intérieur du cercle et qui est à l'extérieur?

$J . R$. Starhawk parle beaucoup de la figure du cercle comme figure nécessaire aux pratiques énergétiques. Elle dit que dans le cercle, «tout le monde se voit». Les ventres, les yeux, les bouches, sont à la même distance les uns des autres et tout le monde voit tout. Il y a ici quelque chose d'ambivalent ; une nécessité de changement, laquelle serait susceptible de rejouer des rapports d'inclusion/exclusion. En même temps, j'ai déjà pratiqué avec des personnes qui ne connaissaient pas cet outil et ça n'a posé aucun problème sur la fertilité de la discussion, la carte n'est qu'un starter pour le récit.

O.N. Le tarot serait un outil artistique plus inclusif, plus horizontal sur le plan des histoires qu'il peut générer.

J.R. C'est intéressant de poser la question. Est-ce que les pratiques divinatoires peuvent constituer un outil qui ouvrirait l'art à quelque chose de plus inclusif, à un langage différent, à une autre manière de communiquer ? Un outil qui nous aiderait à 
sortir de ces novlangues dans lesquelles nous baignons dans le monde de l'art contemporain.

O.N. Le protocole initial engage une forme d'horizontalité, puisque l'outil postule que ta parole (d'artiste) a autant de poids, autant d'importance en termes de mots et de signification que celle du·de la consultant·e. C'est un élément important car ce n'est quasiment - jamais le cas dans les milieux artistiques, traditionnellement régis par une dichotomie entre celui.celle qui sait (l'artiste, le.la critique, le.la théoricien.ne, le.la médiateur.rice, etc.), et celui.celle qui ne sait pas (le-la spectateur.rice). On postule trop rarement que la parole de l'autre, de celui.celle qui reçoit l'art, est importante et signifiante. Dans les pratiques artistiques-ésotériques, cela va même plus loin, puisque c'est le·la consultant e qui construit le sens.

$J . R$. Il y aurait peut-être ici une leçon à tirer ; qu'est-ce que le rituel divinatoire amène dans l'attention portée à l'autre?

O.N. Ce type de dispositif accueille la parole de l'autre, mais aussi son corps, et implique alors une attention au confort, au bien être, à tout ce qui peut guider l'imagination (ce que tu bois, ce que tu manges, comment tu es assise, etc.).

$J . R$. Aussi, dans le contexte des pratiques artistiques divinatoires, la question de la valeur de l'œuvre se déplace. L'œuvre est réussie si l'objet est bien chargé énergétiquement, s'il est issu d'une collaboration heureuse et fertile, si celle-ci a peu d'empreinte carbone. C'est une sensibilité que l'on développe au fur et à mesure. J'ai compris aujourd'hui l'efficacité politique de refuser, dans la pratique artistique, de participer à une économie symptôme d'une logique capitaliste. Les pratiques artistiques-ésotériques permettent ce déplacement, notamment à travers l'attention portée à la provenance des objets, et non à la production de nouveaux objets.

O.N. J'ai observé effectivement que les artistes concernées évoquent souvent le rapport qu'elles entretiennent avec les objets manipulés. D'où viennent les cartes? L'histoire de l'objet participe au rituel et son récit existe parfois en introduction du rituel performatif, comme une amorce, un lancement du protocole.

$J . R$. Les conditions dans lesquelles le rituel se déroule sont aussi importantes. Il y a des lieux artistiques dans lesquels je ne souhaite pas travailler, parce qu'ils sont chargés de mauvaises énergies, par exemple lorsque les conditions de travail des salariée's sont mauvaises, parce que le lieu est dans une logique de surconsommation, parce que les relations hommes-femmes y sont difficiles. L'attention à ces conditions participe à la mise en place d'un système plus vertueux, un système de production d'art plus sain pour l'artiste et le monde.

\section{Le tirage en croix}




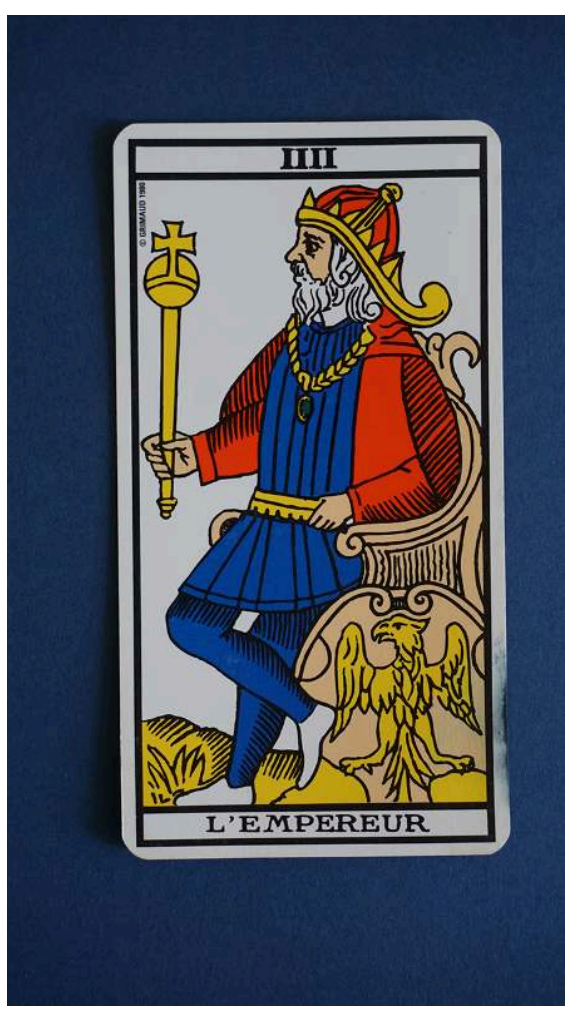

O.N. Je suis étonnée que sorte une figure si forte, si puissante. On pourrait avoir l'impression que ces pratiques restent à la marge, gravitant autour d'un noyau qui serait le «vrai art», mais peut-être participent-elles à la construction de quelque chose de plus grand?

$J . R$. Je vois cette carte comme une blague. On se demande ici dans quelle énergie est l'art actuellement, et, évidemment, c'est un vieux gars bien posé sur ses acquis. Si la papesse est la figure du matriarcat, l'empereur serait celle du patriarcat. L'empereur représente la stabilité matérielle, la puissance. Et en même temps, il n'est pas tout à fait bien assis, on sent que c'est une figure qui peut se laisser déséquilibrer. Peut être a-t-il besoin de se laisser détrôner? C'est une carte ambivalente. Elle a de prime abord quelque chose de l'ordre d'une virilité vulgaire, mais sa posture corporelle raconte quelque chose de plus subtil. Aujourd'hui dans l'art contemporain, il y a bien des concepts obsolètes, moisis, poussiéreux. Dans cette dynamique, je vois des femmes qui détrônent, qui arrivent avec de vraies puissances, avec des alternatives dans les manières de faire, qui ne reprennent pas les codes d'une masculinité forte, mais font autrement. Évidemment lorsqu'on convoque Jodorowski, on en vient à la figure du Père, aux problématiques de construction de la personnalité par rapport à celui-ci. Le tarot de Jodorowsky, s'il reste fondateur, doit aussi être détrôné, et être interrogé sur ces attributs masculins et féminins, afin de se détacher d'une lecture masculiniste.

O.N. L'art serait donc un vieux machin poussiéreux, un père auquel nous devrions rendre des comptes, faire plaisir et, même si les choses sont en train d'évoluer positivement, il subsiste des résidus dans un secteur encore extrêmement patriarcal.

$J . R$. J'ai bon espoir que quelque chose d'autre soit en train d'émerger, qui détrônerait le vieux monde régit par des logiques de domination masculines, mercantiles et matérielles. Si la société française est une société fondamentalement sexiste, 
misogyne, raciste, homophobe, et l'art n'échappe pas à ces règles, il y a aujourd'hui des acteurstrices qui participent à un réel changement.

Deuxième carte : le Monde (les obstacles)

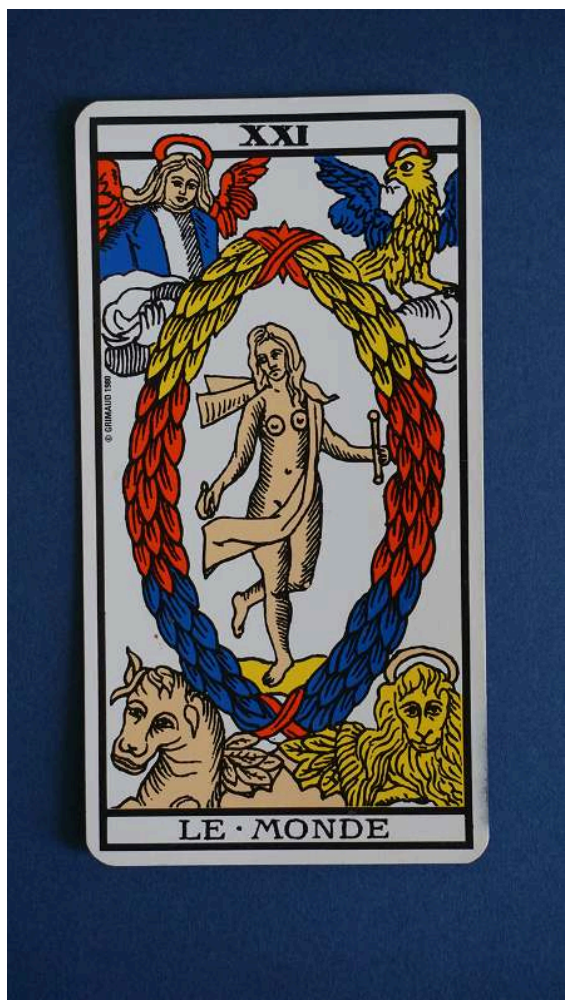

$O . N$. Un personnage féminin au centre. Une chimère, un lion, un cheval, un ange, une couronne. Je pense au cortège dionysiaque avec ses satyres, ses ménades, qui dansent, chantent, en jouant de la musique. Ce tissu qui s'enroule autour du corps féminin me rappelle les représentations antiques des bacchantes, la couronne celle de pampre. II y a chez les ménades comme chez les satyres une ambivalence, d'un côté un aspect festif, joyeux, enthousiasmant, et en même temps un phénomène susceptible de basculer dans la violence. Aussi, il a dans cette carte une intrication femme/nature intéressante, dans laquelle la sexualité a une place importante.

$J . R$. Cette carte, c'est la fin du chemin. Une carte en forme de vagin ; une amande avec une femme nue au milieu. Je me dis qu'elle ne peut pas être un obstacle et qu'elle tombe mal à cet endroit. Elle s'oppose à la première carte; cet empereur avec son trône et son sceptre, et en face une femme nue dans un vagin entourée d'animaux. Peut-être qu'il ne s'agit pas d'un obstacle mais d'un contre pouvoir: un cortège dionysiaque proche de la manifestation interspecies. Nous sommes face à une communauté hybride animal-humain, avec une forte présence du végétal, qui renverse la carte sur un versant heureux. Le monde renvoie au succès collectif; nous avons cheminé, travaillé ensemble, et nous réussissons. Ce n'est pas anodin qu'il s'agisse d'une des rares cartes collectives; face à l'empereur solitaire, se dresse un groupe, et pas n'importe lequel, un groupe interspecies et joyeux.

O.N. II y a aussi l'ange. Pourrait-il·elle être un·e veilleur-se ? Celuilcelle qui empêcherait le basculement négatif? Dans Rêver l'obscur, Starhawk décrit les différentes postures dans les groupes de paroles. Elle parle d'une «guetteuse d'ambiance ». Celle qui est attentive à la circulation des énergies. Un·e personnage qui surveille sans être dans la répression. La figure du·de la veilleur.se, si elle est très présente en danse, dans les pratiques somatiques, 
et aussi dans le bdsm, est beaucoup plus rare dans les arts plastiques. Dans ce contexte, il est plus fréquent que personne ne veille, personne ne s'inquiète pour l'artiste. Néanmoins, j'ai l'impression que cette attention à l'autre est de plus en plus présente, dans les projets artistiques ou de recherche que nous menons ou auxquels nous participons.

$J . R$. Cette question de la veille est aujourd'hui très présente, notamment à travers le langage, sur la question de l'écriture inclusive par exemple, mais aussi sur la question des conditions de travail de l'artiste; plusieurs collectifs prennent en charge cette veille $^{2}$. Il y a aussi une veille collective qui se met en place sur le sexisme, mais encore très largement féminine.

Troisième carte : l'Étoile (conseil)

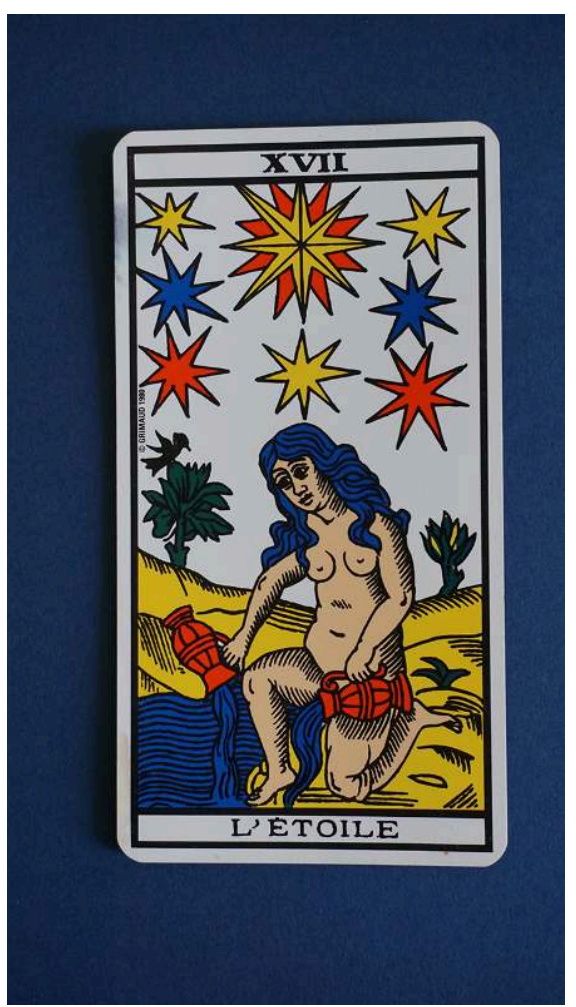

$0 . N$. Une femme verse de l'eau dans une rivière qui se fond avec ses cheveux. Des étoiles, dont une centrale, l'étoile du nord vers laquelle on avance. Je pense à ces pratiques anciennes, qui continuent d'exister dans un coin de notre conscience commune et sont toujours là pour nous guider, à l'image des mouvements alternatifs et de protestation qui réapparaissent de manière cyclique. Quelque chose de plus ancien nous accompagne toujours. La carte renvoie aussi à l'astrologie, mais il ne s'agit pas ici de penser les pratiques divinatoires dans une optique de prescription des destinées, mais plutôt dans les pistes interprétatives qu'elles peuvent suggérer sur les relations entre notre intériorité et notre extériorité.

J.R. Dans l'imaginaire commun, lorsqu'on parle de pratiques divinatoires, on pense au stellaire. La figure est connectée à la terre ; elle est agenouillée sur une terre fertile, et au ciel puisqu'elle touche presque l'étoile. Il y a une impression d'osmose qui se dégage de la carte, comme dans un écosystème vertueux. C'est aussi une carte d'action sur le monde. Placée à l'endroit du conseil, cela peut signifier « faites cela, expérimentez ces alternatives, et de là, un écosystème vertueux se mettra en place ».

O.N. Nous sommes à nouveau dans une combinaison humain/végétal/animal. Comme dans certaines pratiques artistiques (féminines) des années 1970³, on retrouve dans les 
pratiques ésotériques une attention à la nature, aux animaux et à tout ce qui nous dépasse en termes d'énergie. Les objets perdent en importance au profit de la circulation énergétique avec les êtres.

$J . R$. «L'étoile représente une étape où l'on trouve sa place pour agir dans le monde, pour l'embellir et le nourrir à partir d'un lieu que l'on fait sien. » Pensée comme conseil, cela croise ce que dit Donna Haraway lorsqu'elle nous dit de ne pas être ailleurs, mais plutôt de penser à des échelles locales. Il y a urgence à agir. Isabelle Stengers évoque aussi la manière dont on peut se réapproprier une capacité à agir face au sentiment d'impuissance qu'on nous martèle. Nombre de femmes chercheuses, philosophes, nous encouragent à l'action, à tordre le cou à cette idée d'impuissance, à se réapproprier les gestes et les récits. Donna Haraway explique qu'il faut de nouveaux récits heureux, puisqu'à force d'écrire des apocalypses celles-ci sont devenus des réalités. Selon la perspective des narrations spéculatives, on peut dire que l'inverse pourrait fonctionner : si nous racontons des histoires heureuses, à force de spéculer positivement, on pourrait changer l'avenir.

O.N. Notre question s'ouvrirait ainsi à «Que font les pratiques divinatoires à la pensée (et non plus seulement à l'art) à la théorie, à la recherche, etc. ?»

J.R. Dans tous les domaines, la fabulation est fertile. La fiction scientifique est porteuse parce qu'elle en devient philosophique.

$O . N$. Au-delà du contexte artistique, la magie offre de nouvelles manières de formuler nos idées, des modèles de récits, non seulement romanesques, mais aussi politiques. La magie permet de penser le surgissement des possibles, la possibilité d'une autre histoire.

$J . R$. On peut reprendre ici cette corrélation art, magie et politique, puisque pour chacun, il y a des gestes, de la parole, et de l'objet. Entre l'art et la magie, il y a quelque chose de très proche dans l'essence même de leur fonctionnement. Et la politique ressemble à de la sorcellerie, c'est de la parole associée à une gesticulation, à cet objet qu'est la société. C'est aussi ce qui permet de relier actions de militantisme et pratique artistique. Dans le livre de Stengers et Debaise, elle et il expliquent que l'art est un espace pour les gestes spéculatifs (Stengers \& Debaise 2015).

O.N. Certaines artistes auxquelles je me suis intéressée ont en effet repensé fondamentalement la manière dont elles vivaient en termes de consommation, de responsabilité éthique, et/ou sont des personnes engagées sur le plan militant (féminisme, écologie, aide aux migrants, etc.). Qu'est-ce qui se joue dans ce tissage?

J.R. Dans son livre, Marielle Macé part d'une forme - la cabane - (Macé 2019) pour expliquer comment cet objet raconte la nécessité d'accepter la fragilité, d'accepter la mobilité ; on peut bouger de pays, de manière de vivre, de genre, de sexualité. Il y a quelque chose de cet ordre dans l'acceptation des pratiques divinatoires dans le champ de l'art contemporain, qui implique d'accepter de tisser d'autres liens que ceux traditionnellement faits. Dans cette perspective, on s'échappe complètement d'un long héritage conceptuel : Quel est ton discours ? Ton champ référentiel ? Les concepts convoqués? Dans ce type de pratique, au contraire, on accepte le désir, le plaisir, une pratique plus instinctive, viscérale.

O.N. Si on revient à la carte, et à cette eau qui coule, celle-ci me renvoie à l'idée d'accepter dans l'art - l'infiltration, accepter qu'il y ait de la chair, du fragile, de la fissure.

$J . R$. «C'est une carte qui invite à ne pas choisir entre deux options en apparence inconciliables mais à concilier les deux ». 
O.N. Accepter de ne pas être dans une logique schizophrène entre militantisme et pratique artistique, entre réflexion et action, entre théorie et sensible. À nous de revendiquer le droit au mélangé, à la fragilité.

Quatrième carte : le Diable (première synthèse)

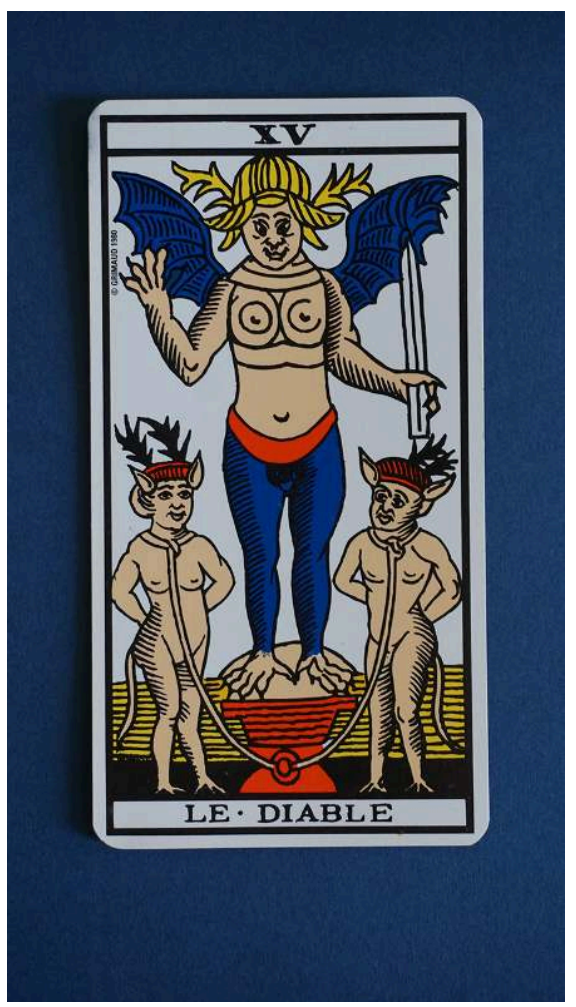

O. N. Un personnage féminin, un·e diable-sse ailé·e, qui convoque la force vive, le désir, le charnel, la sexualité, la sensualité.

J.R. Évidemment, dans le contexte des pratiques divinatoires sorcières, cette carte devait être là. C'est un·e personnage hermaphrodite, et davantage, un'e personnage interspecies. La connotation culturelle négative renvoie à l'obscurité, à nos peurs, et en même temps c'est la carte de la créativité. Lorsque j'ai vu Stengers et Starhawk à la Z.A.D. (de Notre Dame des Landes), elles évoquaient cette idée de faire de l'obscurité quelque chose de fertile. Il ne s'agit pas de se débarrasser de notre colère, nous avons le droit d'être en colère, en lutte. En cela elles rejoignent d'une certaine manière une philosophie de la violence telle que pensée par Elsa Dorlin (Dorlin 2017).

O.N. Nous sommes aujourd'hui dans une situation dramatique sur les plans écologique, politique, social, et c'est une idée séduisante que de penser que de la catastrophe, de l'obscurité puisse naitre une fertilité.

J.R. Qu'est ce qui poussera sur les ruines? Des fleurs ou des champignons ? Il existe un livre sur les champignons et les ruines qui évoque cette fertilité de la moisissure (Lowenhaupt Tsing 2017). Aussi, le·la diable-sse est accompagné·e par deux petit·e·s compagnon'ne's joyeux'ses. Dans la littérature qui mêle magie et politique, la question des alliée'es est importante. 
O.N. Penser les allié.e.s, c'est aussi se donner la possibilité de penser des ennemi.e.s. Dans certains contextes, cela paraitrait presque tabou, alors que se battre « contre » me semble plutôt une idée saine et enthousiasmante.

$J . R$. Je me rappelle de cette discussion que j'avais eu avec ta collègue sur cette pièce "Un bataillon sacré », elle m'avait demandé : « Mais pourquoi un bataillon? ». Parce qu'il y a une bataille à mener. À nous de savoir quelle est la bête à terrasser, à renverser. Je suis en colère, en lutte contre un certain milieu de l'art qui exploite les autres, contre ceux·celles qui veulent régir nos émotions. Cette carte renforce l'idée d'un droit à la colère, à une énergie sombre. Lâchons les chien'ne·s! Détrôner l'empereur demande une énergie viscérale et collective.

Cinquième carte : le Pendu (la somme)

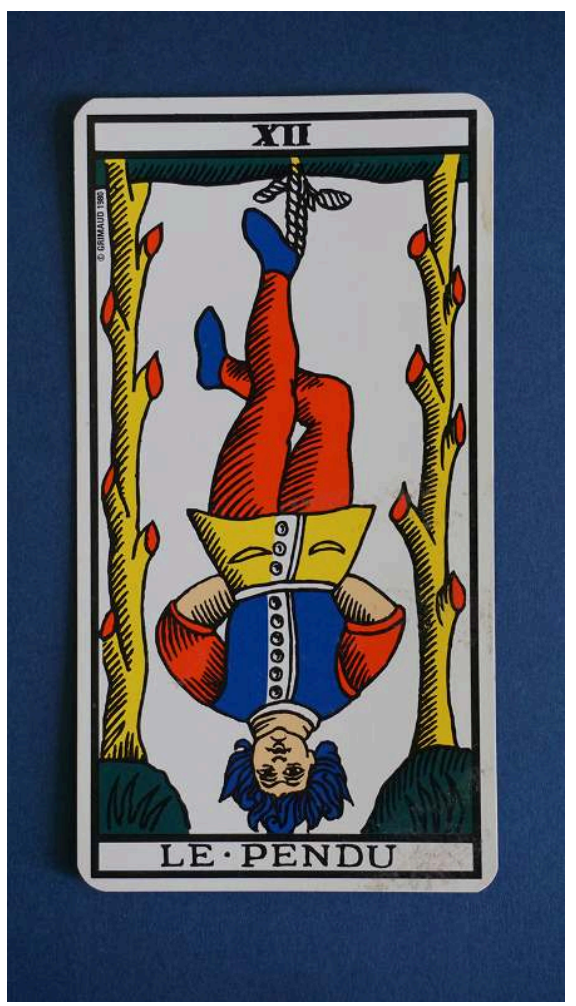

O.N. Une carte qui génère des appréhensions tandis que si on l'observe attentivement, elle n'a rien d'angoissant. II y a quelque chose de l'ordre de la danse, d'une posture très étudiée sur le plan physique, et gracieuse. Selon le sens du regard, la perspective change.

$J . R$. J'aime l'idée qu'il n'y ait pas de "bon sens », de bonne manière de regarder les choses. On retrouve cela dans les pratiques magiques. Le pendu est aussi la carte du don de soi. C'est un tirage très charnel, dans lequel les corps sont engagés, dénudés ou dans des positions très physiques. Et j'ai justement l'impression que l'art contemporain manque cruellement de corps, d'attention portée aux corps, tandis que la pratique magique entraine cette attention.

O.N. Non pas seulement représenter les corps, les présenter comme des objets, mais penser une forme de (ré)-incorporation.

J.R. Dans les pratiques ésotériques comme dans les pratiques protocolaires, cela ne fonctionne que s'il y a un corps. Tu ne peux pas mentir sur ton engagement. Si tu n'es pas engagée, même mentalement, «ça ne marche pas ». 
O.N. Dans un art qui convoque la présence de l'autre, si tu n'es pas engagée, il n'y aura pas non plus d'engagement - corporel et - de la part de l'autre (le.la consultat $e$ ).

J.R. Dans ces pratiques, le rapport à la croyance est important. Cela implique qu'on puisse lâcher prise avec la rationalité, expérimenter la croyance, et observer ce que celle-ci peut produire en nous et autour de nous. Croire aujourd'hui, c'est éminemment politique.

O.N. II ne s'agit pas de renoncer à la rationalisation, mais de prendre en charge ces outils ésotériques qui permettent de nous décaler légèrement, d'adopter un autre point de vue, et ainsi, peut-être, de les transformer en outils d'émancipation.

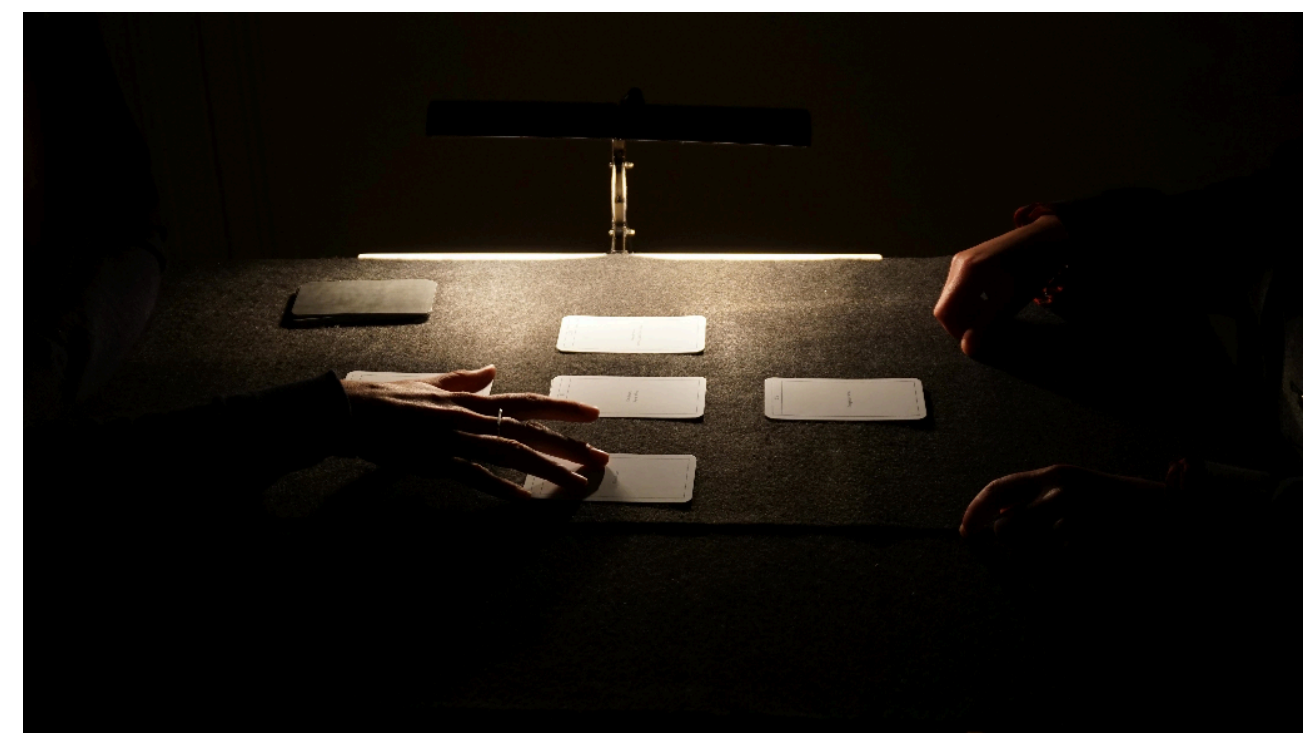

Johanna Rocard, L'avenir entre nos mains/Tarot poétique, dispositif performatif à partir d'un jeu de 22 lames, 2017.

Johanna Rocard

\section{BIBLIOGRAPHIE}

DORLIN, Elsa. 2017. Se défendre. Une philosophie de la violence. Paris : La Découverte.

HARAWAY, Donna J. 2016. Staying with the Trouble. Making Kin in the Chthulucene. Durham: Duke University Press.

JODOROWSKY, Alejandro. 2004. La voie du tarot. Paris : Albin Michel.

LOWENHAUPT TSING, Anna. 2017. Le champignon de la fin du monde. Sur les possibilités de vivre dans les ruines du capitalisme. Paris : La Découverte.

MACE, Marielle. 2019. Nos cabanes. Lagrasse : Verdier.

STARHAWK. 2015 [1982]. Rêver l'obscur. Femmes, magie et politique. Paris : Cambourakis.

STENGERS, Isabelle, DEBAISE, Didier (éd.). 2015. Gestes spéculatifs. Dijon : Les Presses du réel. 


\section{NOTES}

1. Plus d'information sur son travail : https://johannarocard.wixsite.com/travaux

2. Prenons par exemple ici l'important travail mené par le réseau Économie Solidaire de l'Art.

3. Nous pensons ici à des artistes telles Judy Chicago, Anna Halprin, Ana Mendieta, etc.

\section{RÉSUMÉS}

Cet entretien inaugure une série dans le cadre d'une enquête plus vaste menée auprès d'artistes plasticiennes qui utilisent des techniques divinatoires dans leur pratique. Ces interviews sont menées sous la forme de tirages de tarots.

This interview inaugurates a larger investigation about visual female artists who use divination techniques in their practice. These interviews are conducted in the form of tarot readings.

\section{INDEX}

Thèmes : Explorations

Mots-clés : entretien, tarot, art contemporain

Keywords : interview, tarot, contemporary art

\section{AUTEURS}

\section{OPHÉLIE NAESSENS}

Ophélie Naessens est Maitresse de conférences en Arts Plastiques à l'Université de Lorraine. Ses recherches théoriques et artistiques actuelles portent sur les modalités de représentation d'une parole donnée à travers des processus d'enquête et la création d'espaces de parole/espaces d'écoute, ainsi que sur l'échange discursif pensé comme forme artistique (« dialogical art »).

\section{JOHANNA ROCARD}

Johanna Rocard, née en 1985, vit et travaille à Rennes. Elle combine dans son travail des éléments disparates porteur d'une mémoire incertaine et fictionnalisée : reliques de rituels païens, images d'archives de temps révolus ou imaginaires, pierres tombales ou magiques, objets chinés chez le grossiste d'articles de fête. Elle invente un langage archéologique du devenir, témoin d'une société où l'amour est à la fois acte politique et bien de consommation. http:// johannarocard.wixsite.com/travaux/home. 